\title{
Psicología en la Salud: Sociopsicológica o Psicosocial? La Innovación en el Contexto de la Respuesta Brasilera al SIDA
}

\author{
Vera Silvia Facciolla Paiva ${ }^{1}$ \\ Departamento de Psicologia Social e do Trabalho da Universidade Estadual de São Paulo, \\ São Paulo, Brasil
}

\section{Resumen}

Este texto inicia con una revisión crítica de la literatura sobre los usos y el sentido del término psicosocial en periódicos brasileros. Discutirá cómo la concepción brasilera del "modo psicosocial" en la atención al sufrimiento mental, producida desde el movimiento por la reforma sanitaria, contribuyó para constituir un campo de estudios y prácticas innovador, desarrollado en el contexto de la respuesta brasilera al Sida. La perspectiva vulnerabilidad y derechos humanos (V\&DH), poco conocida en la intimidad, basada en los derechos humanos y en la análisis de la vulnerabilidad (social, programática e individual) produjo una vertiente de la psicología social construccionista en la salud que se define como psicosocial y no socio psicológica. Se distingue por abordajes psicosociales de las prácticas en diversos niveles y territorios de atención y prevención. Las técnicas y procesos de trabajo de este abordaje de la vulnerabilidad, destacan la mediación programática y, efectivamente, de la mitigación de la violación de derechos. Inspirada por la hermenéutica y por el construccionismo social, esta vertiente de la psicología social construccionista priorizará la comprensión de la "intersubjetividad en escena" implicada en escenarios socioculturales - en los encuentros programáticos en servicios focalizará escenas cotidianas y de la trayectoria de la persona. La noción de persona, concebida como sujeto de discursos y de derechos, contrasta con la noción de individuo biológico-comportamental de la psicología de la salud que permaneció en otros paradigmas sobre procesos salud-enfermedad. Este artículo ofrecerá referencias para una reflexión crítica sobre el contexto histórico y académico de la producción incluida en este suplemento.

Palabras clave: Psicología social, derechos humanos, vulnerabilidad, constructivismo, promoción de la salud.

\section{Psicologia na Saúde: Sociopsicológica ou Psicossocial? Inovações do Campo no Contexto da Resposta Brasileira à Aids}

\section{Resumo}

Uma revisão crítica da literatura sobre os usos e o sentido do termo psicossocial em periódicos brasileiros inicia este texto. Discutirá, em seguida, como a concepção brasileira do "modo psicossocial" na

\footnotetext{
Endereço para correspondência: Departamento de Psicologia Social e do Trabalho, Instituto de Psicologia, Universidade de São Paulo, Av. Prof. Mello Moares, 1721, Cidade Universitária, São Paulo, SP, Brasil 05508900. E-mail: veroca@usp.br

Agradecemos el apoyo de la Fundación Ford, que nos invitó a sistematizar y, a continuación, a traducir la producción que hace más de dos décadas nos distingue como grupo activo en la respuesta al Sida. Para la producción de este suplemento, la bolsa del CNPq (Consejo Nacional de Desarrollo Científico y Tecnológico), que valoriza mi dedicación a la investigación y permite la coordinación de las actividades de NEPAIDS (Núcleo de Estudios para la Prevención del Sida de la Universidad de São Paulo). Este suplemento no sería posible sin el compañerismo y alegría en el trabajo de los colegas de la red que compone el NEPAIDS, de la equipe del periódico y del trabajo riguroso de los pareceristas.
} 
atenção ao sofrimento mental, produzida no movimento pela reforma sanitária dos anos 1980, contribuiu para constituir um campo de estudos e práticas inovador, desenvolvido centralmente no contexto da resposta brasileira à Aids. Reconhecida internacionalmente, mas pouco conhecida na intimidade, a perspectiva baseada nos direitos humanos e de análise no quadro da vulnerabilidade (social, programática e individual) produziu uma vertente da psicologia social construcionista na saúde que se define como psicossocial e não sociopsicológica. As técnicas e processos de trabalho derivados desta abordagem da vulnerabilidade e dos direitos humanos $(\mathrm{V} \& \mathrm{DH})$ se distinguem pela valorização da mediação programática e, portanto, da ação pela proteção ou para mitigação da violação de direitos. Distinguem-se, ainda, pela abordagem necessariamente psicossocial das práticas nos diversos níveis e territórios de atenção e prevenção. Inspirada pela hermenêutica e pelo construcionismo social, essa vertente da psicologia social construcionista priorizará a compreensão da "intersubjetividade em cena" implicada em cenários socioculturais -nos encontros programáticos em serviços focalizará cenas cotidianas e da trajetória da pessoa. A noção de pessoa, tomada como sujeito de discursos e de direitos, contrasta com a noção de individuo biológico-comportamental da psicologia da saúde que permaneceu nos paradigmas para pensar o processo saúde-doença. Este artigo oferecerá, portanto, referências para uma reflexão crítica sobre o contexto histórico e acadêmico da produção no campo da Aids incluída neste suplemento.

Palavras-chave: Psicologia Social, direitos humanos, vulnerabilidade, construcionismo, promoção da saúde.

\title{
Social Psychology and Health: Socio-Psychological or Psychosocial? Innovation of the Field in the Context of the Brazilian Responses to AIDS
}

\begin{abstract}
A critical review of the literature on the uses and meanings of the expression psychosocial in Brazilian journals opens this text. It will discuss how the Brazilian notion of "psychosocial mode" to organize mental health care, framed within the health reform movements in the $1980 \mathrm{~s}$, contributed to the formation of a field of studies and innovative practices, developed mostly in the context of the Brazilian response to AIDS. Its human rights perspective is unknown in its details as well as its constructionist approach to health psychology defined as psychosocial rather than socio-psychological, distinguished also by a psychosocial approach at the various levels and territories of prevention and care practices. The techniques and work processes derived from a unique Brazilian vulnerability and human rights approach (V\&HR) value the programmatic mediation of the inextricably linked social and individual vulnerability and, therefore, actions for mitigation of rights violations. Strongly inspired by hermeneutics and the social sciences constructivism, this approach to constructionist social psychology prioritize the comprehension of "inter-subjectivity in scene", understood as intrinsically related to socio-cultural scenarios - approached in programmatic encounters at health services through personal trajectory and everyday life scenes. The notion of person, taken as a subject of discourses and holder of rights, contrasts with the notion of a biological-behavioral individual of the health psychology tradition that remained active in other paradigms of health-disease processes. This article will provide references for a critical reflection on the historical and scholarly production in the field of AIDS included in this supplement.
\end{abstract}

Keywords: Social Psychology, human rights, vulnerability, constructionism, health promotion.

Hay un conocimiento que opera en la práctica fundamental para la producción de su renovación. Si de la práctica no nos dedicamos a teorizar, desperdiciamos el trabajo humano de reflexión. La emergencia de la epidemia del SIDA en la década de los 1980s fue, sin duda, 
un estímulo para la innovación de las prácticas, técnicas y teorías implicadas en el desafío de abordar dimensiones sociales y psicosociales en la salud y la enfermedad.

Internacionalmente elogiada por haber ampliado el enfoque individualista, tomando en cuenta el contexto social y la adopción de políticas desde la perspectiva de la protección y promoción de los derechos humanos, la "respuesta brasileña al SIDA", un conjunto de programas y prácticas pioneros organizados desde los 1980s, fue renovadora (Berkman, Garcia, Muñoz-Laboy, Paiva, \& Parker, 2005; Kerrigan et al., 2013; Nunn, da Fonseca, Bastos, \& Gruskin, 2009; Paiva, 2002). Algunos autores hablan de lo cuanto dependía de la coordinación activa entre los investigadores, del gobierno y la sociedad civil que organizaron intervenciones estructurales e institucionales, antes de la existencia del SUS (Sistema Único de Salud), como se puede leer en el artículo sobre la respuesta religiosa en este suplemento. Ha sido una inspiración para los demás países. La producción rigorosa de información precisa que, al mismo tiempo que sigue a través de la ciencia las varias dimensiones de la epidemia, cuenta con la participación de las personas directamente afectadas por el SIDA, algo que la distingue de las respuestas a varias otras enfermedades endémicas en Brasil. Su éxito más notable fue la organización de una red de servicios de salud articulados al nivel intersectorial guiados por los principios del SUS, han ofrecido el acceso gratuito y universal a las pruebas del VIH y el tratamiento integral a las personas diagnosticadas con VIH, así como la prevención centrada en el uso del condón (Berkman et al., 2005; Greco \& Simon, 2007; Malta \& Beyrer, 2013; Paiva, 2002).

Es intuitivo reconocer que estas acciones dependen en gran medida de los enfoques psicosociales que, como veremos, se inspiran en la tradición de las humanidades y de las ciencias sociales aplicadas, especialmente con relación a la enfermedad y la sexualidad y a las prácticas de salud basadas en los derechos humanos (Gruskin \& Tarantola, 2008, 2012; Kalichman \& Diniz, 2009; Kerrigan et al., 2013; Nunn et al., 2009; Paiva, 2002, 2008, 2012a). Como es articulado en varios artículos de este suplemento, las prácticas psicopedagógico y el asesoramiento en las frentes para el SIDA de la respuesta más eficaces en Brasil aseguran la formación continua de los profesionales de diversos sectores (salud, educación, justicia, asistencia social, en las empresas), invistieron para entender y mitigar el proceso de estigmatización y discriminación, valorando las interacciones entre profesional-paciente y servicio-cliente para apoyar la adherencia a la medicación y el uso de condones, con resultados comparables a los países más ricos del hemisferio norte.

Muchas de estas prácticas resultaron de la producción brasileña sobre las dimensiones psicosociales del proceso salud-enfermedad, cuyos autores no siempre son psicólogos. Esta producción teórica y práctica es poco conocida a nivel internacional y merece atención especial por parte de otros campos temáticos y otras disciplinas. La diseminación más organizada de esta producción aumentó la calidad de la respuesta a otros problemas de salud -las enfermedades sexualmente transmisibles, el abuso de drogas y alcohol, el sobrepeso, la tuberculosis y la hepatitis viral- y respondieron al desafío de las prácticas de humanización la salud, en el campo de la salud de las mujeres y los niños.

Este texto proporcionará algunas referencias para una reflexión crítica sobre el contexto histórico de esta producción académica $\mathrm{y}$, en particular, sobre el significado del término psicosocial utilizado de esta manera.

\section{El Uso del Término Psicosocial}

La "(baja) calidad de la atención psicosocial" y la necesidad de que (más) "atención a los usuarios con riesgos psicosociales" 3 son consideraciones frecuentes hechas en textos sobre la atención sanitaria. El los documentos institucionales que organizan programas y políticas gubernamentales de salud, esta expresión indica

2 Psicossocial care es el término más común en Inglés.

3 En varios documentos del Ministerio de Salud de Brasil (Ministério da Saúde, 2008). 
principios generales de acción, en el sentido de defender el principio de atención integral que se centra en y se expande a una clínica tradicionalmente reducida al tratamiento de la enfermedad. En la gran parte de la literatura más académica, pocos definen con precisión el significado del término psicosocial o de los ámbitos psicosociales, los cuales se deben abordar. Aún más raramente, esta literatura se dedica a describir los detalles íntimos de estas prácticas y procesos del trabajo que se podrían adoptaren este sentido.

No es una tarea sencilla: afirmar la dimensión psicosocial envuelve la integración de esferas tratadas por separado históricamente en el entrenamiento profesional-la parte social apartada del individual y la sociedad apartada de la persona. Esto es para tener en cuenta el guion en psico-social ${ }^{4}$, la señal que indica la relación entre las palabras compuestas y abolida en la reforma de la ortografía del portugués. Algunos autores que escriben en inglés en el campo de la psicología defienden el guion. ${ }^{5}$ El guion significaría al mismo tiempo la irreductibilidad de las dimensiones de que es calificado como psicosocial (y viceversa), señalando la imposibilidad de un ámbito existir sin el otro, siendo complementarios. El mantenimiento del guion se traduce a la insistencia en alguna diferencia, "una diferencia que no puede ser superada y que continuamente invita a la participación y la exploración" (Hogget, 2008, p. 13).

¿Cómo aparece el término psicosocial en portugués y en las bases de datos más accesibles de la literatura disponible en formato electrónico? Aunque se recuperó en numerosos artícu-

4 El debate sobre el guión se inició en el Departamento de Psicología Social de la Universidad de São Paulo por Belinda Mandelbaum, ${ }^{\text {Luiz Galeão }}$ y Nelson Luiz da Silva Júnior, en un documento interno que escrito en 2011, no fue publicado, a quienes les debo la inspiración.

5 Nota del Traductor: El guion és utilizado cuando la palabra es usada como adjetivo (sociopsychological en ingles pero sociopsicología en español) La separación por el espacio entre las palabras es usada cuando se refiere al sustantivo (en todas los idiomas, por ejemplo social psychology, psicología social, etc.). los, libros y tesis ${ }^{6}$, psicosocial no figura como descriptor, se encuentra sólo como un adjetivo asociado con otras palabras clave. No es un descriptor en la BVS-Psi ULAPSI (Biblioteca de Salud-Psicología Virtual), la referencia de América Latina con sede en Brasil dedicada al desarrollo profesional de los psicólogos y al avance de la investigación en psicología. No aparece como terminología utilizada en las ciencias de la salud (DeCS), utilizada en SciELO. Cuando es utilizada la fuente de la APA (American Psychological Association), nos encontramos con el término psicosocial como una calificación de la "rehabilitación" (cognitiva, del abuso de drogas, física) o asociado con "el desarrollo psicosocial" que se puede utilizar como sinónimo de "desarrollo social" cuando hace referencia a la obra de Erick Erikson. Para pensaren el carácter de la producción brasileña, cuando la fuente del término es el $\mathrm{SBD}^{7}$ brasileño, lo encontramos asociado con las palabras clave "Centro de Atención Psicosocial" o "intervención psicosocial”. En la terminología de la salud, ni como un adjetivo puede ser localizado.

En la literatura disponible en SciELO que revisamos $^{8}$, encontramos muchos sentidos de lo que se define como el ámbito psicosocial, o simplemente cualificado como psicosocial, y la mayoría no ofrece una definición precisa del significado del término. Este se utiliza para definir

6 En agosto de 2013, se recuperó con el scielo.br el término psicosocial en 841 artículos, 390 en la BVS y en más de 218 tesis y 40 libros.

7 Servicio de Biblioteca y Documentación, sede del BSV-Psi ULAPSI en el Instituto de Psicología de la Universidad de São Paulo.

8 Esta es una revisión sobre el tema del tipo de narrativo (Rother, 2007), realizada en artículos científicos recuperados en la búsqueda a través de SciELO, utilizando los términos "psicosocial" o "psicosociales" y el año de publicación (20002012). Indicado por los objetivos propuestos en este artículo, una reflexión crítica sobre el uso del campo de la salud psicosocial, se eligió la base de datos SciELO porque el la se concentra en revistas que pasan por una evaluación más rigurosa y sistemática para incluir un número bien indicativo y representante de los periódicos brasileños de más alta calificación. 
una dimensión que no depende únicamente de la persona (al estudiar el individuo) o que califica fenómenos entendidos como "sociales" $y$ "psicológicos" al mismo tiempo, significando simplemente que no podrían ser apenas psicológicos o sociales. Al abordar el proceso de la enfermedad-salud, muchos de los artículos disponibles usan la definición de salud que viene del discurso de la Organización Mundial de la Salud (OMS) como "el bienestar físico, mental, social y espiritual".

Por ejemplo, en los procesos de trabajo que describen el rendimiento de un historial médic (anamnese) ${ }^{9}$, en el ámbito psicosocial están las recomendaciones para tener en cuenta el contexto de la vida del paciente e incluso una evaluación profesional mediante el lugar en el que reside la persona (sugerencia común de la práctica en el sistema de revisión judicial)a cambio de un enfoque basado en el proceso "psicodiagnóstico"10 clásico, que es guiado por la descripción de la dinámica intrapsíquica universal y sin contexto. ${ }^{11}$ Este tipo de evaluación, que incluiría la situación de vida de las personas, es llamada una "evaluación psicosocial".

Muchos textos hablan de la "integración psicosocial" en referencia a un trabajo de apoyo y rehabilitación para las personas adaptando a una nueva condición de vida. El proceso de trabajo exitoso en la integración evita el aislamiento, el sufrimiento y la exclusión social. Los términos "ajuste psicosocial" y "rehabilitación psicosocial" se asocian a esta literatura.

Cuando el tema son los niños, niñas y adolescentes hay un sentido de adaptación asociada a la noción de "desarrollo psicosocial" que se utiliza en el sentido de adaptarse a lo que se espera de cada etapa de la vida, las etapas tratadas como esenciales y universales. En consecuencia, no mencionan el contexto, el género,

9 Historiava desde los síntomas iniciales hasta el momento de la observación clínica, se realiza basado en la memoria del paciente.

10 Los diagnósticos de los trastornos mentales que usando métodos o pruebas psicológicas.

11 Es interesante notar que se trata de una tecnología de evaluación psicosocial que no se enseña en las universidades principales. la clase social y la tradición cultural en la que se incluyen los niños y los jóvenes. Esta comprensión del proceso de desarrollo se define con mayor claridad cuando se inspira en diferentes psicoanálisis. La mayoría de los artículos, sin embargo, no hace referencia a ningún autor o conceptualización del desarrollo psicosocial. Un grupo más pequeño de los artículos se refieren a documentos del Ministerio de Salud de Brasil, como aquellos relacionados con la organización de equipos multidisciplinarios en la comunidad (para profesionales de salud mental, enfermeras, ginecología y otras clínicas) para la atención integral de salud de los adolescentes que incluye la noción de contexto como sinónimo de "medio ambiente".

"Se entiende por desarrollo psicosocial la capacidad humana de adquisición progresiva de interactuar con su medio ambiente" (Ministério da Saúde, 1993, p. 37).

Como en este documento, sin embargo, aunque se menciona la comunidad y el medio ambiente, la mayoría de los textos mantienen la noción de "maduración biopsicosocial" y se refieren al "riesgo de anormalidad" producido por el contexto, la mayoría entre los pobres "en las zonas rurales y en las afueras los grandes centros urbanos" (Ministério da Saúde, 1993, p. 18).

Por otro lado, hubo un aumento en el número de textos disponibles en SciELO que considera los conceptos psicológicos de "apoyo social", "redes sociales" y "relaciones institucionales", en contextos como la familia, el mundo del trabajo, las organizaciones de la sociedad civil y en el gobierno. Al referirse a la comunidad, muchas veces se refieren a un territorio que delimita una identidad, o a segmentos de la sociedad agrupados por afinidad psicosocial, presunta o supuesta - como en una favela o entre homosexuales, grupos religiosos, personas que viven con el VIH.

En este conjunto de estudios realizados en los últimos años, se añaden aquellos que incluyen la categoría psicosocial para definir las relaciones de poder y la ciudadanía, en especial las "relaciones de género", de la clase y, más raramente, las relaciones étnicas y raciales. Las referencias a la clase o la pobreza, o al "género" se incluyen en el ámbito considerado psicosocial, 
inspirado en la tradición de los estudios culturales y la perspectiva construccionista de género. Esta tradición, al discutir la diferencia encarnada en ciertos grupos sociales (mujeres y hombres, pobres y ricos) y al pensar sobre estados de desigualdad social, afirma que éstos no pueden ser explicados singularmente por la "naturaleza" o por los extensos procesos sociales inevitables. Las estructuras y relaciones sociales socializan las personas y son históricamente establecidas, pero la desigualdad ni es inevitable ni tolerable. ${ }^{12}$ En este conjunto de textos, sin embargo, son poco los que desarrollan la clase o el género como categorías de análisis, en su organización y en la narrativa, como en las propuestas de Brah (2006) o Scott (1988/1995).

Diversos fenómenos se definen como de la dimensión psicosocial en los diferentes enfoques: sentimientos, emociones, actitudes y prácticas, discursos extraídos de las relatos de las personas o analizadas sobre la base de los documentos, dinámica relacionales, de la resistencia y la resiliencia, escenas y contextos. La expresión que más utilizan los autores más influyentes en el campo construccionista es la de "aspectos psicosociales". Expresión utilizada en los dos estudios sobre desigualdad, cuando tratan del mundo del trabajo y de las organizaciones califican de psicosocial la relación de individuo-grupo/instituciones psicosociales, o individuo-sociedad.

Es innegable que, aún sin definir con más detalle el sentido práctico y nuevas técnicas de los conceptos menos universalistas y adaptativos, se ha considerado cada vez más el nivel simbólico y cultural como algo más expresivo de la dimensión psicosocial en la interacción con otros "aspectos" o "factores" situados en la organización social $y$ el en sujeto/individuo/persona. Un número creciente de artículos también hablan

12 Entre ellos, muchos autores latinoamericanos que citan Gergen y Ibañez, Berger e Luckmann, o se inspiran George Meady Goffman y otros autores que abordan los ámbitos psicosociales inspiradas en el interaccionismo simbólico y el pragmatismo en estudios culturales, estudios feministas y de la sexualidad. Han leído Foucault, el marxismo, la fenomenología y la filosofía de Gadamer, Habermas y Ricouer. de las "formas de ver el mundo" del sujeto/individuo/persona, y de la producción de "sentidos" y el aspecto "discursivo" en las interacciones sociales, interpersonales y en las instituciones.

El término sujeto se utiliza a menudo, pero casi siempre como sinónimo de individuo o persona sin utilizar la palabra con un significado teórico claramente definido. En los modelos que tratan la dimensión psicosocial como una expresión de la asociación de "factores", el término individuo es preferido; en los individuos, la diversidad simbólica y cultural se reconoce en las variables que definen diferentes "percepciones", "creencias", "actitudes", “ opiniones", "conocimiento", "normas", e "identidades".

Especialmente interesante para este texto, el uso repetido del término psicosocial como referencia al "modo psicosocial" para organizar la asistencia a los enfermos mentales. Así se expresa en la terminología incluida por el SBD mencionado anteriormente - Centro de Apoyo Psicosocial. Como uno de los resultados del movimiento de la Reforma Sanitaria que concibió el SUS, el uso de la término psicosocial expresa un concepto de atención de la salud mental relacionada con la organización de la atención en la comunidad (no en el hospital), y se refiere al concepto de una "clínica ampliada" y a los derechos humanos, y en particular a las nociones de la "autonomía" y la "dignidad" incluidas en el preámbulo de la Declaración de los Derechos Humanos. El modo psicosocial, en su definición de atención, busca profundizar la práctica de la "integridad" en el cuidado de las personas que sufren de enfermedades mentales y sus diversas "necesidades" - a los cuales también se refieren como derechos; amplia el cuidado anteriormente reducido al tratamiento del problema definido por una nosología o taxonomía patológica. Hay muchas referencias en SciELO a los Centros de Atención Psicosocial (CAPS, en algunos lugares se llaman Núcleos de Atención Psicosociales/ NAPS). Aún en este grupo de textos, las prácticas llevadas a cabo basadas en el "modo psicosocial" son descritas muy poco, y el término aparece con frecuencia para subrayar que "no todo depende de la persona", y en oposición al "modelo biomédico" genérico. 
También es interesante comparar estos datos con lo que se observa en la literatura internacional. Hace algunos años, en busca del significado de la atención psicosocial ("psychosocial care") solicitamos al director del área científica de la Psicología Aplicada de la APA (Asociación Americana de Psicología) que, reconociendo la polisemia de expresión y la falta de una definición precisa, ofreció una explicación tomada de la literatura del área de servicios sociales, con la intención de presentar "un contexto para el término":

El término psicosocial describe una constelación de necesidades sociales, emocionales y de salud mental, y el cuidado que se ofrece al atenderlas. Un concepto más amplio y relacionado es el de la calidad de vida, desde la perspectiva de la persona, que incluye su experiencia en el hogar y no sólo la atención médica. La atención psicosociales multifacético y a que además de los asistentes sociales, otros profesionales de la salud pueden contribuir para satisfacer las necesidades psicosociales y mejorar la calidad de vida que también dependen de los procesos y el medio ambiente en general en el hogar de la persona. (S. R. Johnson, comunicación personal, 21 marzo, 2007) $)^{13}$

Nuestra revisión crítica de la literatura de la década $2000-2009^{14}$ encontró la expresión "atención psicosocial" en el sentido de: (a) la atención a problemas emocionales o a sufrimientos mentales que limitan el funcionamiento social de los enfermos, (b) la necesidad de organizar o apoyar a las personas para lidiar con los problemas de la vida cotidiana cuya origen es social- como la falta de dinero, de vivienda, de higiene y el acceso a los insumos necesarios para el cuidado de la salud, entre otros, (c) los cuida-

13 Stephanie R. Johnson PhD. Director of Applied Psychological Science. Science Directorate American Psychological Association. Comunicación con Eliana Zucchi por e-mail, 21 de marzo de 2007.

14 Investigación en MEDLINE y SciELO de 20002009, usando o término "psychosocial care" y "atenção psicossocial" realizada con apoyo de Eliana Zuchi y Bruna Robba Lara. dos paliativos para mantener la calidad de vida de los enfermos terminales.

Muy rara en la literatura internacional y común en la literatura brasileña, se puede encontrar también la noción de asistencia psicosocial de atención asociada a (d) asistencia que incluye a la comunidad como parte de la responsabilidad de organizar el cuidado de los enfermos o las personas con discapacidad. En la literatura que no-brasileña, textos basados en esta perspectiva casi siempre discuten iniciativas emprendidas, desarrolladas e implementadas en los países en desarrollo o en comunidades empobrecidas o estigmatizados (como los homosexuales), especialmente en los países Africanos y más afectados por el SIDA, donde, por la ausencia de la atención a la salud organizado y tratamientos médicos, cuenta con la comunidad para ayudar a los enfermos y discapacitados con los cuidados paliativos.

En los campo de la medicina, enfermería y psicología de la salud, comúnmente se utiliza la categoría "factores psicosociales" para constructos concebidos con base en análisis estadísticas da asociación entre respuestas a cuestionarios. A partir de preguntas y respuestas cuantificables, las "condiciones de vida" y el contexto son transformados en variables "socioeconómicas" (educación, ingresos, religión, color/raza) o "sociodemográficas" (como datos sobre los hijos y relaciones conyugales). La mayoría de estos estudios describen la asociación de los factores psicosociales (bajo conocimiento, por ejemplo) como constituyentes de la vida de las personas que sufren de alguna "enfermedad" o como efecto psicosocial, esperado, de la enfermedad (aislamiento). El conocimiento mediador elegido es la epidemiología del riesgo. Algunos grupos de personas consideradas "en alto riesgo" después de ser tipificados en estos análisis corresponden a "grupos de riesgo". Los factores psicosociales se asocian con la enfermedad y afirman la relevancia de ellos en el proceso de la salud-enfermedad. De estos estudios, sin embargo, no se deriva necesariamente una innovación significativa para la práctica de atención. Cuando lo hacen, con mayor frecuencia los autores indican que los pacientes cambien sus actitudes, una orientaci- 
ón que profesionales incluyen en su práctica de "prescripción" y permanece técnica individualista: se pone en el individual la responsabilidad de mantener buenos comportamientos y lidiar con el contexto.

Los estudios sobre la salud sexual tienen una teoría más sofisticada para interpretarla asociación entre diferentes factores psicosociales. Los resultados de investigaciones clínicas y de comportamiento epidemiológicos e han discutido más directamente a través teorías de carácter universalista e generalizable para cualquier contexto, influenciadas en gran parte por la sexología, cuyos antepasadosson Freud, Reich, Kinsey y Masters y Johnson (Paiva, 2008).

“. . . de facto el desempleo puede causar problemas de erección" (Abdo, Oliveira, Moreira, Abdo, \& Fittipaldi, 2005).

Cuando el enfoque es constructivista social la sexualidad es interpretada considerando las interacciones sociales ${ }^{15}$, las relaciones de poder y los derechos humanos, - derechos sexuales y reproductivos. Raramente no se derivan políticas y acciones programáticas, así como las agendas de investigación para comprender e mitigar el sexismo, la homofobia y la desigualdad de género, como en la literatura sobre la salud de la mujer y el SIDA. Interpretaciones y prácticas en esta perspectiva no serán generalizables para cualquier contexto. Fue con esta perspectiva que a lo largo de la respuesta al SIDA se discutieron los problemas que la noción de "grupos de

15 John Gagnon, el científico social que se incluye como antecesor del construccionismo y da un enfoque dramatúrgico a lo psicosocial, que muchos autores adoptan en este suplemento. Interaccionista y heredero del pragmatismo y la Escuela de Chicago, su uso de la "carrera" y "trayectoria" le ayudó a definir con Simon (Simon \& Gagnon, 1969) y antes de Foucault, el sexo como una actividad social como cualquier otro y la forma en que los discursos sociales sobre el sexo reemplazan a noción de impulso. La conducta sexual en términos físicos y simbólicos, puede expresar otros intereses (trabajo, política, religión) que no tienen prioridad en la explicación causal. Es decir, se invierte Freud al afirmar que el sexo puede significar casi todo en la vida social y el sexo no tenía prioridad en la explicación causal (Gagnon, 2006, p. 406). riesgo" causó en para el enfrentamiento a la epidemia y a las vidas de las personas afectadas. La banalización de la noción de grupos de riesgo fomenta el estigma y la discriminación de los segmentos identificados como de riesgo, además de incitar la negación del problema en las personas que no se identifican con este estigma, sin ofrecer técnicas y prácticas o procedimientos de trabajo apropiados, adecuados y de acuerdo con los principios del SUS (Ayres, Calazans, Saletti, \& França, 2006).

La noción del riesgo psicosocial, sin embargo, aún prevalece en el campo de la psicología de la salud, en los hospitales y en los enfoques mediados por la epidemiología. Predomina esta noción aun cuando se substituye el término "riesgo" por "vulnerabilidad" sin el sentido del concepto cambiar, como veremos en seguida.

\section{La Dimensión Psicosocial en la Organización de las Prácticas de Salud}

En el campo de la organización de las prácticas de salud, de manera similar, como veremos en esta sección, palabras compuestas resumen el debate sobre la forma de concebir el proceso de la producción de la salud y de la enfermedad, que produjo tres movimientos paradigmáticos en la segunda mitad del siglo XX. Las palabras compuestas también expresan una dirección de acciones que no han sido descritas con precisión. $\mathrm{Su}$ definición como biopsicosocial, como notamos en el caso de la salud de los adolescentes, tiene como objetivo ampliar la perspectiva exclusivamente biológica o biomédico y requiere acciones multiprofesionales.

Como se discutió en Ayres, Paiva y França (2010/2012), el marco conceptual que, en la década de 1950, postuló por primera vez la superación de los límites disciplinares entre la intervención social en salud pública y la práctica clínica, entre el tratamiento y la prevención, se conoce como el modelo de la "historia natural de la enfermedad" (HNE). Leavell y Clark (1958) describieron períodos de una "historia natural de la enfermedad" (HNE) que requeriría una intervención interdisciplinar en las múlti- 
ples causas considerando los tres niveles de prevención (NP). Para este modelo conocido como el HNE-NP, acciones de prevención primaria evitan la exposición a la enfermedad durante el período definido como pre-patogénico (por ejemplo, con vacunas y con el uso condones). En el período patogénico, cuando la enfermedad ha comenzado, la prevención secundaria (a través de la detección precoz y la intervención clínica apropiada) impide la progresión de la enfermedad y de su transmisión, favoreciendo mejores resultados individuales y colectivos; la prevención terciaria (a través de la rehabilitación y la continuación del tratamiento) buscaría la mejor calidad de vida posible en situaciones de consecuencias inevitables y enfermedad crónica. En este modelo, la epidemiología de riesgo surgió como una de las principales formas de articular conocimiento en este esfuerzo interdisciplinario.

El segundo movimiento estableció la «Nue-

va Promoción de la Salud" (NPS), siguiendo varias conferencias internacionales en los años 1970 que reforzaran la centralidad de las prácticas de prevención y de atención primaria, cuestionando la centralidad del hospital como el foco de la acción política y programática para la salud. Los determinantes sociales de la salud y las ciencias sociales ganaron importancia en este movimiento, que concibió mejorar las condiciones de la salud individual y colectiva basada en principios que validaron inclusión del derecho a la asistencia sanitaria universal en la Constitución de 1988 en Brasil. El NPS afirmó, al mismo tiempo, una concepción integral de la salud (física, mental, social y espiritual), la acción intersectorial, la participación en la transformación de situaciones sociales y acciones multiestratégicas que promovieran la equidad y la sostenibilidad de las iniciativas de salud, y la participación de los usuarios de servicios. ${ }^{16}$ Todos estos son principios del SUS brasileño que la organización de la respuesta brasileña al SIDA buscó implementar.

Fueron introducidas, desde el NPS, algunas prácticas para la manipulación de "variables" sociales. La psicología que es parte de este movi-

16 Como resultado de la Reforma Sanitarista. miento también identificó aspectos psicosociales en la cadena de múltiples causas responsables por la enfermedad. Muchas de las prácticas en psicología de la salud fueron constituidas en Brasil en este esfuerzo por la integralidad, y los psicólogos que hablaron con la clínica médica e interactuaron en las prácticas generalmente definidas por la "perspectiva biopsicosocial" produjeron nuevos modalidades técnicas y procedimientos. F. M. Santos y Vilela (2009) definen estos ajustes s como «colectivos de pensamiento» y nombraron los más estructurados en los hospitales generales: la Psicología de la Salud, la Psicología Médica, la Psicología del Hospital y Salud Mental que, en el cuidado de la salud, son principalmente basados en fundamentos psicosomáticos, la referencia teórica de psicoanalítica y la psicología existencial.

Muchos autores en estas tradiciones han teorizado el vivir con el SIDA, o interpretaron la relación entre los estilos de vida y la prevención de la enfermedad y la infección por el VIH de acuerdo con modelos sociopsicológicos (socio-psychological) aplicados a la salud. ${ }^{17}$ En el caso de la respuesta al SIDA, estas psicologías estructuraron la práctica en los servicios de "salud mental" especializados para las personas que viven con el VIH. Como se discutió en Paiva (2012b), antes de la producción de remedios contra la reproducción del VIH, la literatura internacional sobre las prácticas de servicios para los enfermos se limitó a las investigaciones sobre el estrés y el afrontamiento, los cuales investigaron asociaciones estadísticamente significativas entre las experiencias de la vida cotidiana y las enfermedades, y las formas de afrontar la enfermedad. Más adelante y especialmente para pensar la prevención, la literatura sociopsicológica introdujo analices basados en variables sociales (etnia, educación, lugar de residencia, género, identidad sexual y religiosa, principalmente). Dos saberes han mediado estas prácticas sociop-

17 En este último caso, Bandura, Proshaska, Lazarus y Folkman, entre otros, son los autores más conocidos (Tunala, 2012), que siguen siendo más citados en la literatura internacional llamada "psicología de la salud". 
sicológicas dentro del NPS: la tradición epidemiológica y la psicología de comportamiento y socio-cognitiva.

Otros enfoques psicosociales dedicados a encontrar instrumentos para practicar el acogimiento y el cuidado más allá de la llamada "salud mental" tuvieron más dificultades en el establecerse. Alternativas se desarrollaron con mayor facilidad en el campo de la prevención y de la atención básica de la salud, donde las prácticas eran menos consolidadas por el equipo tradicional de los psicólogos y habían más incentivos para trabajos interdisciplinares. En el caso del SIDA, otros profesionales (enfermeras, trabajadores sociales, nutricionistas, educadores, además de los médicos y paramédicos) siempre actuaron enfocando aspectos psicosociales en el aconsejaría pos-prueba, la adherencia al uso de condones y medicamentos, y varias otras actividades psicoeducativas desarrolladas en la atención básica y en los servicios especializados.

La alternativa de una referencia más instrumental para la práctica surgió fuertemente con el desarrollo de un tercer paradigma para pensar en el proceso de la salud-enfermedad: el marco de la vulnerabilidad y de los derechos humanos (V\&DH), sistematizado y validado internacionalmente precisamente en la construcción de la respuesta al SIDA en la década de 1990 (Gruskin \& Tarantola, 2012; Mann \& Tarantola, 1996; Mann, Tarantola, \& Netter, 1992). El marco de la V\&DH amplió significativamente las perspectivas biopsicológicas y socio-psicológica, y creció con la Nueva Promoción de la Salud en el sentido de incorporar los determinantes sociales de la salud para luego pensar en la vulnerabilidad individual y social al encuadrar la enfermedad como indisolublemente integrada en una dimensión programática como la Figura 1 intenta ilustrar-dimensión que se realiza en el enfoque en los derechos humanos (Gruskin \& Tarantola, 2012; Mann, Gruskin, Grodin, \& Annas, 1999).

En Brasil, la perspectiva de los derechos humanos fue expandida: los planos individuales, sociales y programáticos han sido diseñados como los planos de intersubjetividad, como en la esfera dinámica de la vida cotidiana, de ciu- dadanía y el sujeto de derechos (Ayres, Paiva, \& França, 2010/2012; Paiva, 2012a). Este marco produjo un enfoque psicosocial construccionista de la salud y sustituyó la noción del individuo concebido como un conjunto de factores bio-socio-psicológicos, del trabajo original de Mann y sus colegas, para plantearla persona en su contexto, concebida como sujeto de la saludy del derecho a la salud (Paiva, 1996, 2002, 2008, 2012a, 2012b). Reconociendo los avances que la introducción de las variables sociales en psicología de la salud significó para el necesario diálogo interdisciplinario, la unidad central o portal para acercarse a la dimensión psicosocial no es el "individuo", una entidad que la tradición de la social-psychology concibió como un constructo que resulta de factores socio-cognitivo-comportamentales. La unidad de análisis elegido fue la escena de interacción intersubjetiva (en los servicios, la vida social y cotidiana, en la trayectoria personal). Todos los planos de la vulnerabilidad son mutuamente implicados como la figura indica.

Una primera novedad a ser subrayada en este tercer movimiento (V\&DH), en contraste con la NPS, es el peso dado a la expansión político-programática, un determinante social e intersubjetivo, como un mediador de la vulnerabilidad social e individual. En pocas palabras, la vulnerabilidad a la enfermedad de las personas que pertenecen a los segmentos sociales más vulnerables y marginalizados, (por ejemplo, los más pobres) disminuí o aumenta en la presencia o ausencia de programas de salud (por ejemplo, la aplicación de la vacuna, la existencia de acciones de saneamiento básico, acciones para prevenir el SIDA, el acceso a la atención médica y al tratamiento). Este enfoque explica algunos de los éxitos de Brasil en la reducción de la propagación del SIDA, incluso en la década de 1990, antes de disminuir la pobreza, el sexismo, la homofobia y el estigma asociado con la enfermedad, o de resolver los problemas de abuso de drogas y el acceso universal a la salud integral. Los programas contra el SIDA desarrollaron iniciativas innovadoras que abordaron técnicamente $\mathrm{y}$ programáticamente estos determinantes, $\mathrm{y}$ los resultados fueron sorprendentes. 


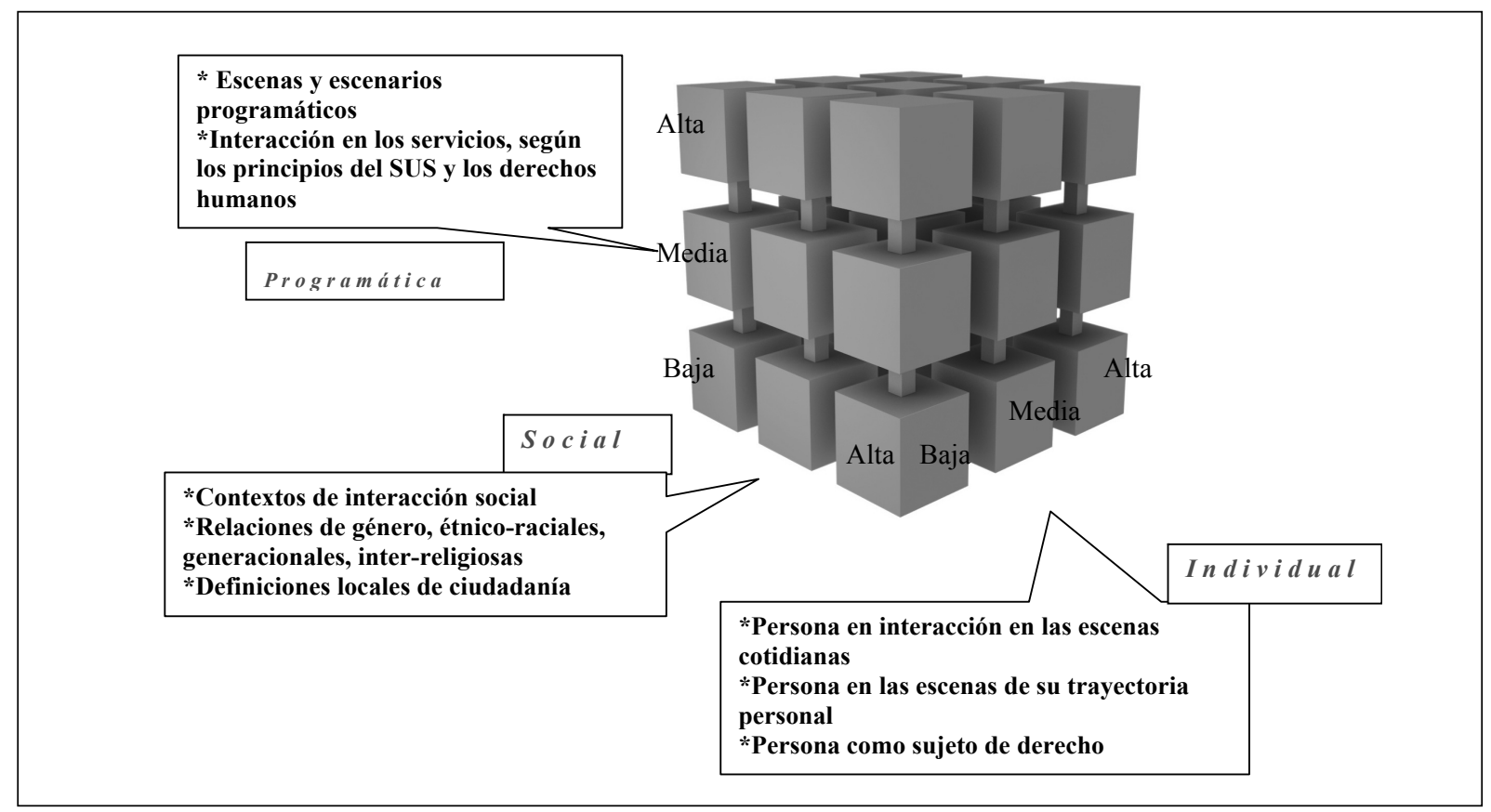

Figura 1. Interdependencia de las dimensiones social, programática e individual de la vulnerabilidad, concebidas como intersubjetividad.

Esta mediación programática de muestra de forma emblemática que la negligencia o violación mayor de los derechos humanos lleva a la probabilidad más alta de enfermedad, y por lo contrario, cuanto más el esfuerzo sea por promover y proteger los derechos _ a la salud integral, los derechos sexuales y reproductivos, en acciones contra la discriminación, la seguridad alimentaria, vivienda digna, trabajo digno, entre otros modos _ más existe la probabilidad de supervivencia y menos la oportunidad de infectarse. Se asume en este marco, como principio, que toda sociedad debe proteger y promover los derechos para promover la salud, y que sus gobiernos (nacionales y locales) pueden ser considerado responsables en caso de negligencia o violación de derechos (Gruskin \& Tarantola, 2012). El uso de este principio por parte de activistas y profesionales de la salud en el SUS y en condiciones de responder a las demandas del movimiento social, fue notable en la producción de la respuesta brasileña al SIDA.

Se trata de superar la individualización, así como no colocar la culpa solamente en la "víctima" o la persona responsable por su descuido y por los factores contextuales asociados a su enfermedad. Programas y prácticas basadas en un análisis de la vulnerabilidad a la enfermedad dependen necesariamente de la acción interactiva de los diversos conocimientos (de clínicas diversas, de la epidemiología, de las ciencias humanas y sociales), y de una práctica desde la perspectiva de los derechos humanos (desde la gestión hasta la atención), o sea, que el sistema no discrimine, que garantice el acceso universal a servicios de salud, de su aceptabilidad y calidad, y de la participación del usuario.

Pensando más allá de la llamada dimensión individual de la vulnerabilidad, por lo tanto, rápidamente abandonamos en Brasil la socio psicología de la obra original de Mann et al. (1992, 1997). Presente en la literatura norte-americana y europea, como se podría esperar ${ }^{18}$, el enfoque socio psicológico aún sigue siendo hegemónicamente diseminada por las instituciones de las Naciones Unidas, las cuales adoptaron el marco de V\&DH. Aquí, la tradición latinoamericana de la pedagogía, la psicoterapia y el teatro del oprimido (Boal, 1975; Freire, 1968/1973; Moffat, 1987; Oliveira \& Araujo, 2012) inspiró una psicología dedicada a la acción social y comunitaria, que algunos llaman "intervención

18 Nuestra producción autóctona en América Latina tiene poca fuerza de separación, sobre todo porque no escribimos (y por lo tanto pensamos) en Inglés. 
psicosocial", definida como palabra clave por el SBD y no por la APA, como se mencionó anteriormente", con implicaciones en la construcción de ciudadanía post-colonial y post-dictadura. Esta misma tradición produjo la "atención psicosocial" que fue introducida en el SUS.

Este fue el contexto en que surgió el movimiento de la teoría práctica que se dedica a desarrollar un enfoque psicosocial en la salud en el cuadro de la V\&DH (Paiva, 2012b). Se construye en éste, como en otros aspectos del construccionismo, una alternativa a la noción de individuos "objetos" de intervención, modelados, y de la prescripción de la educación bancaria o de la predicación moralizante (Paiva, 1996, 2002, 2007). Preferimos el término persona utilizado en el lenguaje de los derechos, así como en las definiciones del sujeto al que se refiere el campo diverso construccionista (Cañón, 2008).

En este aspecto del enfoque psicosocial en el contexto de los Derechos Humanos y la Vulnerabilidad $^{19}$, el sujeto se entiende (a) como sujeto de discursos, los cuales cada persona encuentra en su trayectoria y proceso de socialización, un proceso que no la domina fundamentalmente; las personas, como la antropología y la psicología social sobre la salud han indicado durante décadas, viviendo para reinterpretar discursos en diversos contextos de sus vidas. En su vida cotidiana, por ejemplo, las personas son "sujetos sexuales" para volver a dibujar, interpretar, los distintos discursos sobre el sexo (la prevención, la religión, los medios de comunicación) para llevar a cabo sus sexualidades en cada ocasión y escena (Paiva, 1996, 2007). Como "sujeto religiosos", interpretan dogmas y discursos religioso adaptándolos a su religiosidad personal (García, Muñoz-Laboy, Almeida, \& Parker, 2009; Silva, Santos, Licciardi, \&

19 Cuando entendemos e interpretamos los datos de diversos conocimientos acerca de la vulnerabilidad de una persona o un grupo social a un problema de saluda menudo digo que estamos en el marco de la V\&DH. Cuando estamos diseñando y planificando la práctica, los principios de los enfoques de derechos humanos se destacan y por lo general invierten las siglas DH\&V.
Paiva, 2008). Como (b) sujeto de derechos y en solidaridad con los demás se puede decidir por la expansión y la especificación de nuevos derechos. Es como sujeto de derechos que el usuario de servicios debe ser entendido en la intimidad de las prácticas y técnicas del enfoque psicosocial: como sujetos en los lugares donde reciben cuidado, sujetos de aprendizaje en solidaridad con los demás, como agentes de la emancipación de la opresión psicosocial y de la vulnerabilidad a enfermarse (Paiva, 2002, 2008, 2012a; Seffner, Paiva, \& Pupo, 2012).

La producción latinoamericana de este enfoque de la psicología social de la salud, por lo tanto, lleva el mismo tipo de reflexión crítica que expresa Frosh (2013), quien también discute los límites de las teorías llamadas sociopsicológicas (socio-psychological) e individualizadoras. Frosh subraya que la sociopsicología investiga las cogniciones y comportamientos en los contextos sociales que operan con la noción del individuo como un hecho, no como una construcción, apoyando la división intelectual del trabajo que se separa diferentes disciplinas que estudian la sociedad (lo social antes del guión) de los que estudian al individuo (psico después del guión). Frosh entre otros, le da valor a la intersección. Otro desafío adicional, en su opinión, es superar la idea de que haya algo «objetivo», el individuo, que puede ser estudiado desde una posición «neutra», sin responder al contexto y a las ideas que lo habitan. El autor atribuye el «asujetamiento» al trabajo de la sociedad, en la formulación de Butler (1997), y apoya la posibilidad de ser «sujeto» con poder para actuar en el mundo. Esta es una expansión de la inspiración europea en Freud y Marx para concebir e investigar un sujeto que es a la vez social y psicológico para así, como dueño de agencia y de vida interior, superar la represión.

En Brasil, desde la década de 1990, esta misma crítica se informó diversos aspectos de la psicología constructivista social, incluso en la salud (Spink, 2013). Como se podría esperar, muchos autores que participaron en la respuesta al SIDA en algún momento de su carrera se juntaron con este punto de vista. Todos reconocemos la centralidad de los estudios de género y 
de la sexualidad, que extendieron su legitimidad precisamente en la respuesta al SIDA y a las exigencias de la organización de la salud integral de la mujer. ${ }^{20}$

Colocando nuestra crítica construccionista en el campo de los derechos humanos y de la vulnerabilidad, parece significativo que socio viene antes de psicológico en los modelos de los estudios de psicología de la salud o la epidemiología sociopsicológica: como la práctica de mediación, éstos se resumen en las asociaciones entre factores obtenidos en muestras grandes, a partir de datos agregados y al nivel de la población que tienen sentido sólo para los estudios sobre tendencias y probabilidades macro-sociales, cuando recurren a otros conocimientos. Indebidamente predominantes, por lo tanto, las prácticas y medidas preventivas encentraran estas tendencias de esta misma manera, hasta al tratar de entender la dinámica en la vida cotidiana de las personas. Al hacer esto, no alcanzan ofrecer los recursos técnicos para renovar definitivamente las prácticas.

Como si fuera un conocimiento mediador y único, el análisis de riesgos coloniza indebidamente otros campos del conocimiento, tales como el cuidado de las personas y la prevención, con recetas de "estilos de vida adecuados" a los

20 El Brasil organiza hace 18 años el mayor encuentro que debate exclusivamente la categoría género ("Fazendo Gênero") que aglomera, literalmente, millares de investigadores. América Latina es co-productora del gran cambio epistemológico producido por los estudios de género y sexualidade construccionista, inspiración fundamental de esa vertiente de la psicología hace más de dos décadas. Como Terezita de Barbieri sintetizó, la producción de la categoría género del análisis es "una ruptura epistemológica, tal vez la más importante en las ciencias sociales en los últimos 20 años, porque reconoce una dimensión de la desigualdad social hasta entonces no abordada, sometida a la dimensión económica, en las teorías de clase y en las teorías de estratificación social" (Barbieri, 1991). Los autores que trabajan en el campo del Sida valoran los estudios de gênero mucho antes que la obra de Butler fuera conocida y tuvo también aquellos que, de algún modo fueron inspirados, como Ella, por la tradición psicoanalítica. individuos "genéricos" en su bio-psico/comportamiento, personas-típicas, que no existen en la realidad. Un ejemplo de esta colonización ineficazes la insistencia en la predicación universal sobre el uso de condones, que más de 20 años después ha mostrado sus límites. El movimiento social del SIDA en Brasil insistió desde el año 2011 que en la $4^{\text {a }}$ década de la epidemia tendríamos que singularizar la prevención, pensando en las relaciones de género (las mujeres no usan preservativos disponibles y dependen de técnicas de conversación) y que cada persona tiene el derecho de utilizar todos los recursos disponibles en el contexto de sus interacciones sexuales, incluyendo la prevención biomédica (ver http:// oquenostiraosono.tumblr.com/home).

Como sintetizaron recientemente Kippax, Stephenson, Parker y Aggleton (2013), las concepciones sobre el plan del individuo utilizado en los campos de la epidemiología del riesgo y la sociopsicología, y por cierta versión del marco de la V\&DH de la Organización Mundial de la Salud ${ }^{21}$, olvida: (a) que las acciones de las personas no son simples productos de sus características o de sus decisiones racionales, pero si están implicados en redes de sentido y significados, (b) que cuando el plan de la persona se reduce a un cierto comportamiento (relaciones sexuales sin uso del condón) desaparecen los significados diferentes que las prácticas asumen a través del tiempo por la misma persona en diversas lugares que ocupa y ocasiones que vive (como un cónyuge o amante; por el amor o en situaciones de abuso);(c) que la normatividad cultural y las prácticas sociales constantemente producen el individuo y sus prácticas.

Kippax y sus colegas (2013) sustituyen la noción del individuo como centro de la prevención por la focalización en la comunidad, por la acción colectiva y la interacción entre los grupos y redes de personas. Ellos argumentan que los cambios sociales y de comportamiento que se han articulado en la comunidad transformaron en el curso de la epidemia del SIDA. La centralidad de la noción de movimiento comunitario para la

21 Expresado en el documento (Joint United Nations Program on HIV/AIDS [UNAIDS], 2011). 
promoción de la salud se resalta en forma en que el condón fue introducido como un artefacto de la anticoncepción y se ha convertido en la norma entre los hombres homosexuales que, en la década de 1980, se organizaron en un movimiento social de resistencia a la epidemia. Ellos argumentan que la movilización basada en la solidaridad y en el cuidado comunitario produjo el acceso universal al tratamiento antirretroviral en la respuesta brasileña al SIDA, como es discutido en varios artículos de este suplemento.

Por otro lado, el desafío señalado por Frosh, por Kippax y otros construccionistas parece ser casi insuperable para cualquier persona que trabaje en el campo de la salud, donde por ambición de objetividad y el manejo clínico o quirúrgico del cuerpo individual, extraído de su contexto cultural, han tenido un efecto práctico incuestionable. Considere el tratamiento para el VIH en una persona con SIDA, por ejemplo: ¡en sus protocolos se resumen un conjunto de hipótesis teóricas probadas a través de técnicas y prácticas que se basan en un manejo riguroso de sustancias, productos, por cuerpos extraídos de sus contextos sociales para ser probados y tratados; aquí o en cualquier otro continente, resulta en eficacia indiscutible! ¡Los antirretrovirales controlan la infección por el VIH en cualquier parte del planeta! Mientras los espacios de práctica multidisciplinaria y multisectorial necesitan de una concepción alternativa de este constructo del individuo biológico-comportamental para que se adopten enfoques tecnocientíficos que reconozcan que los remedios son efectivos cuando se garantizan el acceso a ellos y se mantiene la adherencia al tratamiento, como este suplemento discutirá.

Esta misma discusión realizada en el campo de la sexualidad también parecía insuperable al inicio de la epidemia del SIDA, cuando la literatura global y las psicologías exportados a varias otras disciplinas académicas (a la educación y a la salud) expresaban la hegemonía de la tradición sexológica que concibe un sexobiopsicosocial, con dinámica igual y desarrollo psicosexual universal. Este enfoque todavía se dedica a revelar lo que es psicosexualmente normal y las verdades esenciales sobre el sexo obtenidas a través de encuestas clínicas o poblacional es que derivan prácticas directamente para la clínica de los trastornos sexuales. Para la prevención de la transmisión sexual del VIH tener cierta eficacia, la respuesta al SIDA también señalo los límites de esta concepción sobre las relaciones sexuales y sobre las técnicas utilizadas para derivarlas intervenciones para garantizar el "desarrollo psicosexual normal" o "saludable", como en los programas para adolescentes que fueron mencionados anteriormente, un tema que se desarrolló en otros textos (Paiva, 2008, 2012b; Paiva, Ayres, \& França, 2004).

El reconocimiento de los límites de estos esencialismos para responder al SIDA permitió el crecimiento de puntos de vista alternativos. Sobre todo en el hemisferio sur o en las áreas del hemisferio norte donde era evidente que un sinergismo de plagas (Parker \& de Camargo, 2000) produce desigualdad social basada en relaciones de dominación de clase, en el sexismo, en el racismo y en la naturalización de genocidios, que sustentaba la explosión en el número de casos de SIDA. La epidemia descontrolada exigió que la reflexión crítica reformulara los protocolos para la práctica rápidamente que, en aquel momento, se desarrollaron con la referencia ético-política de los derechos humanos; y permitió la renovación técnica (del conocimiento operativo que es de carácter normativo, prescriptivo y metodológico) y teórica (del conocimiento de carácter analítico y comprensivo).

¡En otras palabras, cuando hay una sinergia de las desigualdades, la estigmatización, y la mayor vulnerabilidad a la enfermedad, un guión es poco! Los que están envueltos en la promoción de la salud necesitaban las flechas de interacción y de la integración, la sinergia de conocimientos, y las intervenciones en diferentes dimensiones institucionales, estructurales y políticas. La aparición de la epidemia del SIDA, en todas partes, intensificó la necesidad de actuar de forma sincrónica, dinámica y simultáneamente en todas las dimensiones que afectan el proceso de la enfermedad. En este sentido, es fundamental un diálogo interdisciplinario mientras que los espacios integrados se estructuran para acción y para reconstruir las prácticas derivadas de este 
diálogo para que los beneficiaran los segmentos tradicionalmente incluidos en la vida social a través de la discriminación (Ayres, Paiva, \& Buchalla, 2012).

Por lo tanto, una segunda novedad del marco de la V\&DH, que también distingue su enfoque psicosocial de otros construccionistas en la psicología social, es su ambición de producir encuentros fértiles entre los diversos conocimientos. El crecimiento del enfoque hermenéutico incluido en la versión brasileña del marco de la V\&DH lo hace diferente. La hermenéutica en el sentido filosófico de la obra de Gadamer (1997), y no sólo en el sentido metodológico, presentó una perspectiva de la relación entre el yo-otro, no sólo en sus aspectos cognitivos, pero también en sus aspectos éticas, morales, políticos y estéticos (Ayres, 2007, 2008). Al lidiar con a la vulnerabilidad abordando las dimensiones individuales y sociales, y por su abordaje programático, la hermenéutica evita un objetivismo que promueve la reducción de lo social a estilos de vida, la dicotomía entre lo individual y lo colectivo, entre lo biológico y lo social; evita también el sentido puramente instrumental de los aspectos estructurales y la politización que sigue externa de los aspectos tecno científicos. Los aspectos socio-estructurales deben participar, estar presentes, en la práctica (el conocimiento basado en las relaciones interpersonales, intersubjetivas, y que es responsivo e individualizado). Esta hermenéutica politiza la teoría y la técnica en la intimidad de sus protocolos. ${ }^{22}$

Esta hermenéutica produjo la idea del Cuidado en el marco de la vulnerabilidad (Ayres, 2004a, 2004b) y valora la importancia del cuidado adoptado en diversos campos del conocimiento y de en práctica en la salud (Pinheiro \& Lopes, 2010; Pinheiro \& Mattos, 2006). Motivado por diferentes rutas y experiencias, el enfoque del movimiento de la V\&DH de esta perspectiva filosófica por varios autores brasileños en campos como la medicina, la nutrición, la salud de las mujeres, y de la enfermería, permitió una la producción teoría-técnica sobre lo psicosocial

22 Sobre estas definiciones de teoría, técnica e prática vea Pupo (2012) que debe llamar la atención para la importancia y pertinencia de esta perspectiva.

El objetivismo, las dicotomías y lo estructural entendido como una barrera, así como la política tratada como factor externo, todavía persistieron, aunque haya sido una consecuencia no-intencional, en los discursos y propuestas de la NPS y en las versiones de la vulnerabilidad criticadas por Kippax y colegas (2013). En Brasil, la pedagogía de Paulo Freire ya había superado esta externalización en el campo de la educación, con su pedagogía contra la colonización que también inspiró conceptos de la promoción de la salud en todo el mundo. La inclusión de la hermenéutica en Brasil fortaleció la opción por el uso de enfoques multiculturales y emancipadores al abordar los Derechos Humanos, propuesta en la hermenéutica diatópica de Boaventura Souza Santos (2003) - una manera de operar en el marco de los derechos que se caracteriza por una preocupación anticolonial, similar a la posición de Freire (Paiva, 2012a).

Esta segunda novedad, la perspectiva hermenéutica, subraya la centralidad de la intersubjetividad para pensar sobre la salud en los niveles individuales, sociales y programáticos (como se muestra en la Figura 1) y acentúa la reflexión crítica sobre individualismo. Debido al sentido fuerte del diálogo en la perspectiva hermenéutica, como para Gadamer, es la "fusión de horizontes" (Ayres, 2007), el pensamiento crítico se realizará con la participación directa de los usuarios de los servicios, en los lugares donde se reciben cuidados, y en cualquier plano institucional y político. Enfoques psicosociales están involucrados en la producción de las prácticas del cuidado, de la prevención y de la educación, así como de la programación y gestión de la salud. Se puede recuperar de las intervenciones estructurales su dimensión intersubjetiva: en lugar culpar las huellas macrosociales de la desigualdad, nos lleva a entender el lado concreto de las relaciones de género y raciales, de clase, y la generación en la vida cotidiana, en la forma en que se experiencia en cada territorio y por cada persona, que también encontramos en las prácticas de salud y en cómo se llevan a cabo. 
Por último, la perspectiva de la salud psicosocial usada a través de este marco brasilero de la V\&DH no ignora la centralidad de la persona, entendida como sujetos en interacción con otros y como sujeto en relación a los derechos humanos. Como un sujeto en relación, cada persona puede tener la experiencia de enfermedad y protegerse de ella, lo que envuelve su constitución física y al modo personal de ejercer su cotidiano, como parte de una comunidad. Como sujeto dueño de derechos, puede reivindicarlos, o como agente comunitario o del estado, en solidaridad puede promover y proteger los derechos de otras personas que ve como co-ciudadanos. Comunidades no existen apenas como una noción abstracta definida por normas o referencias culturales. Además de ser encarnadas en la gente, dependen de las personas que son agentes institucionales para legitimar cotidianamente y durante la socialización de las nuevas generaciones, para reproducirlas. O por el contrario, las personas pueden inspirar y organizar la acción y la transformación comunitaria como líderes de los movimientos sociales. ${ }^{23}$

Esta perspectiva y forma de tratar la salud usando este marco de los derechos humanos y de la vulnerabilidad, por lo tanto, son psicosociales e no socio-psicológicos.

A través de esta interpretación, la vulnerabilidad a la enfermedad siempre pertenecerá a una persona (una mujer) - no a un grupo de riesgo (prostitutas) o a una comunidad (un territorio, o un grupo solidario que comparte una identidad) - en cualquier determinada situación social (vivida por la persona como un escena dinámica

23 La noción de la "encarnación” es usada en el mismo sentido es la noción de "movimientos de salud encarnados" donde se describen los movimientos de saluden los Estados Unidos (Brown et al., 2004). Los autores sostienen que estos movimientos presentan el cuerpo biológico en los movimientos sociales en los relatos de sus experiencias de sufrimiento y abandono, especialmente de su experiencia emocional y no sólo cognitiva; interrogan la medicina hegemónica y las ciencias; incluyen activistas en colaboración con científicos y profesionales para el acceso al tratamiento de la salud, la prevención, la investigación y la financiación. con un escenario sociocultural), donde se garantizan sus derechos - o son violados y tratados con negligencia. La vulnerabilidad a un problema de salud será peor o mejor dependiendo de la presencia o ausencia de un programa (de prevención, de apoyo, de cuidado de su salud). ¡La mayoría de las mujeres brasileras que viven con el VIH _ que tuvieron, en promedio, sólo una o dos parejas en la vida- o una proporción las prostitutas, negligenciadas en sus derechos y sin acceso a programas de prevención ejemplifican esta perspectiva!

\section{El Proceso de Renovación de las Prácticas se debe Atravesar hasta Renovar la Teoría}

Las contribuciones de las ciencias sociales, de la historia y de la filosofía han sido fundamentales para el diseño de nuevas prácticas y para la innovación teórica producida en medio de la respuesta brasileña al SIDA. En el caso de la psicología, esta producción de una psicología social más politizada ${ }^{24}$ en la salud puede informar las prácticas que, a partir de los saberes clínicos, se enfocan en lo intersubjetivo y producen los colectivos multiprofesionales dedicados a la salud del individuo y del colectivo en el SUS. Ha sido largo el camino para la validación práctica, intensa la ruta en que las prácticas y sus conocimientos específicos, sus principios, fueran aplicados sabiamente en acciones concretas en el trabajo cotidiano para cuidar de las personas que viven con el SIDA o de las poblaciones socialmente vulnerables. Lo que el marco de la vulnerabilidad nos enseñó es que no hay una Historia Natural de la Enfermedad, sino una Historia Social de la Enfermedad, no sólo porque la enfermedad tiene aspectos sociales e históricos, pero también porque la historia de la enfermedad se cuenta a través de escenas sociales (Ayres, Pai-

24 Farr (1998) comenta acerca de una psicología social sociológica (en contraste con la psicología social psicológica). Aqui estamos pensando en una psicología social politizada en el sentido que se dedica a pensar los poderes, la desigualdad y la intimidad de las políticas públicas. 
va, \& França, 2010/2012), como algunos artículos de este suplemento ilustran.

En el caso del SIDA, la respuesta brasileña necesitaba ser articulada en las prácticas de salud de un sistema universal y que sólo comenzó a ser construido cuando comenzó la epidemia. Paralelamente a la aparición de la epidemia, para tener en cuenta el compromiso de universalidad, equidad e integridad, el Programa de Atención Integral a la Salud de las Mujeres (PAISM) ya es una innovación, superando el guión en salud "materna-infantil" usando otros términos: "mujeres" e "integral". La perspectiva feminista y Marxista-construccionista encontrada en las ciencias sociales, la educación, y el campo de la salud sexual y reproductiva que hoy marcan el movimiento de humanización en las prácticas de salud de Brasil, se remonta a la década de 1980 . Añadido a este movimiento, como inspiración de la respuesta al SIDA, la producción latinoamericana de la psicología social comunitaria, del oprimido e da la intervención psicosocial, dialogan con el sentimiento anti-psiquiátrico, institucionalizado en Brasil como la Reforma Psiquiátrica y de Lucha Contra el Manicomio, y produjo el "modo psicosocial" en la salud mental.

Sólo recientemente este esfuerzo de reflexión crítica y la sistematización de las prácticas requeridas por la organización del SUS y, más recientemente, un Sistema Único de Asistencia Social (SUAS) comienzan a resultar en textos escritos que reflejan una importante renovación teórica. Esta producción se nota especialmente en la segunda década del siglo XXI. Se necesita coraje para transformar el pensamiento y las prácticas colectivas en contribuciones teóricas para apoyar la formación disciplinar de psicólogos brasileños para las generaciones futuras, sin que tengan que reinventar la rueda cada vez, con base en las escuelas dogmáticas basadas en tal o cual autor de otro siglo, o en sociopsicologías producidas en otros contextos sociales.

¡Esperamos que este suplemento ayude a estimular otros, de diferentes ámbitos y temas, con el mismo espíritu de innovación teórica, técnica y práctica!

\section{Referencias}

Abdo, C. H., Oliveira, W. M. de, Jr., Moreira, E., Jr., Abdo, J. A., \& Fittipaldi, J. A. S. (2005). The impact of psychosocial factors on the risk of erectile dysfunction and inhibition of sexual desire in a sample of the Brazilian population. São Paulo Medical Journal, 123(1). Retrieved June 17, 2009, from <http://www.scielo.br/ scielo.php?script $=$ sci_arttext\&pid $=$ S 1516 $31802005000100003 \& \operatorname{lng}=$ en\&nrm=iso

Ayres, J. R. (2004a). Cuidado e reconstrução das práticas de saúde. Interface: Comunicação, Saúde e Educação, 8(14), 73-92.

Ayres, J. R. (2004b). O cuidado, os modos de ser (do) humano e as práticas de saúde. Saúde e Sociedade, 13(3), 16-29.

Ayres, J. R. (2007). Uma concepção hermenêutica de saúde. Physis, 17, 43-62.

Ayres, J. R. (2008). Para comprender el sentido práctico de las acciones de salud: contribuciones de la herméutica filosófica. Salud Colectiva, 4, 159172.

Ayres, J. R., Calazans, G., Saletti, H., Filho, \& França, I., Jr. (2006). Risco, vulnerabilidade e práticas de prevenção e promoção de saúde. In G. W. S. Campos, M. C. S. Minayo, M. Akerman, M. Drumond Jr., \& Y. M. Carvalho (Orgs.), Tratado de Saúde Coletiva (pp. 375-417). São Paulo, SP: Hucitec.

Ayres, J. R., Paiva, V., \& Buchalla, C. M. (2012). Direitos Humanos e Vulnerabilidade na Prevenção e Promoção da Saúde: Uma introdução. In V. Paiva, J. R. Ayres, \& C. M. Buchalla (Orgs.), Coletânea: Vulnerabilidade e Direitos Humanos. Prevenção e promoção da saúde: Vol. 1. Da doença à cidadania (pp. 9-22). Curitiba, PR: Juruá.

Ayres, J. R., Paiva, V., \& Franca, I., Jr. (2012). Conceitos e práticas de prevenção: Da História Natural da Doença ao Quadro da Vulnerabilidade e Direitos Humanos. In V. Paiva, J. R. Ayres, \& C. M. Buchalla (Orgs.), Coletânea: Vulnerabilidade e Direitos Humanos. Prevenção e promoção da saúde: Vol. 1. Da doença à cidadania (pp. 71-94). Curitiba, PR: Juruá. (Reproduzido a partir de Routledge Handbook of Global Public Health, Vol. 1, pp. 98-107, de R. Parker \& M. Sommer, Eds., 2010, London: Routledge) 
Barbieri, T. (1991). Sobre la categoría género. Una introducción teórico-metodológica. In S. Azerêdo \& V. Stolcke (Coords.), Direitos reprodutivos (pp. 25-45). São Paulo, SP: Fundação Carlos Chagas.

Berkman, A., Garcia, J., Muñoz-Laboy, M., Paiva, V., \& Parker, R. (2005). A critical analysis of the Brazilian response to HIV/AIDS: Lessons learned for controlling and mitigating the epidemic in developing countries. American Journal of Public Health, 95(7).

Boal, A. (1975). Teatro do oprimido e outras poéticas políticas [Theatre of the Oppressed]. Rio de Janeiro, RJ: Civilização Brasileira.

Brah, A. (2006, jan./jun.). Diferença, diversidade, diferenciação. Cadernos Pagu, (26), 329-376.

Brown, P., Zavestoski, S., McCormick, S., Mayer, B., Morello-Frosch, R., \& Gasior Altman, R. G. (2004, January). Embodied health movements: New approaches to social movements in health Sociology. Health \& Illness, 26(1), 50-80.

Butler, J. (1997). The Psychic life of Power: Theories in subjection. Stanford, CA: Stanford University Press.

Cañón, O. E. (2008). Las huellas del sujeto en narrativas de autores construccionistas [Subject's tracks in the narratives of constructionista authors]. Revista Diversitas - Perspectivas em Psicologia, 4(2), 245-257.

Farr, R. (1998). As raizes da Psicologia Social Moderna. Petrópolis, RJ: Vozes.

Freire, P. (1973). Pedagogia do Oprimido. Rio de Janeiro, RJ: Paz e Terra. (Original publicado em 1968)

Frosh, S. (2013). Psychosocial Theory. In T. Teo (Ed.), Encyclopedia of Critical Psychology. New York: Springer.

Gadamer, H. G. (1997). Verdade e método: Traços fundamentais de uma hermenêutica filosófica. Petrópolis, RJ: Vozes.

Gagnon, J. (2006). Uma interpretação do desejo: Ensaios sobre o estudo da sexualidade. Rio de Janeiro, RJ: Garamond.

García, J., Muñoz-Laboy, M., Almeida, V., \& Parker, R. (2009). Local impacts of religious discourses on rights to express same-sex sexual desires in peri-urban Rio de Janeiro. Sexuality Research \& Social Policy, 6(3), 44-60.
Greco, D. B., \& Simão, M. (2007, July 21). Brazilian policy of universal access to AIDS treatment: Sustainability challenges and perspectives. AIDS (Suppl. 4), 37-45.

Gruskin, S., \& Tarantola, D. (2008). Universal Access to HIV prevention, treatment and care: Assessing the inclusion of human rights in international and national strategic plans. AIDS, 22(Suppl. 2), 123-32.

Gruskin, S., \& Tarantola, D. (2012). Um panorama sobre saúde e direitos humanos. In V. Paiva, J. R. Ayres, \& C. M. Buchalla (Orgs.), Coletânea: Vulnerabilidade e Direitos Humanos. Prevenção e promoção da saúde: Vol. 1. Da doença à cidadania (pp. 23-41). Curitiba, PR: Juruá.

Hogget, P. (2008). What's in a Hyphen? Reconstructing psychosocial studies. London: Palgrave.

Joint United Nations Program on HIV/AIDS. (2011, October). UNAIDS Terminology Guidelines. Retrieved October, 2011, from http://www.unaids. org/en/media/unaids/contentassets/documents/ unaidspublication/2011/JC2118 terminologyguidelines_en.pdf

Kalichman, A., \& Diniz, S. (2009). AIDS treatment in Brazil: What kind of evidence do we need? The Lancet, 374(969), 1066.

Kerrigan, D., Wirtz, A., Baral, S., Decker, M., Murray, L., Poteat, T., \& Beyrer, C. (2013). The Global HIV Epidemics among Sex Workers. Washington, DC: World Bank.

Kippax, S., Stephenson, N., Parker, R. G., \& Aggleton, P. (2013, June 13). Between Individual Agency and Structure in HIV Prevention: Understanding the Middle Ground of Social Practice. American Journal of Public Health, 103(8), 1367-1375.

Leavell, H. R., \& Clark, E. G. (1958). Textbook of Preventive Medicine. New York: McGraw-Hill.

Malta, M., \& Beyrer, C. (2013). The HIV epidemic and human rights violations in Brazil. Journal of the International AIDS Society, 16, 18817. Retrieved from http://www.jiasociety.org/index. php/jias/article/view/18817

Mann, J., Gruskin, S., Grodin, M., \& Annas, G. (Eds.). (1999). Health and human rights: $A$ reader. New York: Routledge.

Mann, J., \& Tarantola, D. J. (Eds.). (1996). AIDS in the world II. New York: Oxford University Press. 
Mann, J., Tarantola, D. J., \& Netter, T. W. (Eds.). (1992). AIDS in the world. Cambridge, MA: Havard Universty Press.

Mann, J., Tarantola, D. J., \& Netter, T. W. (1997). A AIDS no mundo. Rio de Janeiro, RJ: Relume Dumará

Ministério da Saúde. (1993). Normas de atenção à saúde integral de adolescente: Vol. 1. Diretrizes gerais para atendimento de adolescentes. Acompanhamento do crescimento e desenvolvimento. Distúrbios da puberdade. Desenvolvimento psicológico do adolescente. Brasília, DF: Autor.

Ministério da Saúde. (2008). Portaria $n^{\circ} 154$. Cria os Núcleos de Apoio à Saúde da Família/NASF. Brasília, DF: Autor.

Moffat, A. (1987). Psicoterapia do Oprimido. São Paulo, SP: Cortez.

Nunn, A. S., da Fonseca, E. M., Bastos, F. I., \& Gruskin, S. (2009). AIDS treatment in Brazil: Impacts and challenges. Health Affairs, 28(4), 1103-1113.

Oliveira, E. C., \& Araújo, M. F. (2012). Aproximações do Teatro do Oprimido com a Psicologia e o Psicodrama. Psicologia: Ciência E Profissão, $32(2), 340-355$.

Paiva, V. (1996). Sexualidades adolescentes: Escolaridade e gênero, e o sujeito sexual. In R. Parker \& R. Barbosa (Orgs.), Sexualidades Brasileiras (Vol. 1, pp. 213-224). Rio de Janeiro, RJ: Relume-Dumará.

Paiva, V. (2002). Sem mágicas soluções: A prevenção e o cuidado em HIV/AIDS e o processo de emancipação psicossocial. Interface: Comunicação, Saúde e Educação, 6(11), 25-38.

Paiva, V. (2007). Gendered scripts and the sexual scene: Promoting sexual subjects among Brazilian Teenagers. In Culture, Society and Sexuality. $A$ reader $\left(2^{\text {nd }}\right.$ ed.). New York: Routledge.

Paiva, V. (2008). A psicologia redescobrirá a sexualidade? Psicologia em Estudo, 13, 641-651.

Paiva, V. (2012a). Cenas da Vida Cotidiana: Metodologia para compreender e reduzir a vulnerabilidade na perspectiva dos Direitos Humanos. In V. Paiva, J. R. Ayres, \& C. M. Buchalla (Orgs.), Coletânea: Vulnerabilidade e Direitos Humanos. Prevenção e promoção da saúde: Vol. 1. Da doença à cidadania (pp. 165-208). Curitiba, PR: Juruá.
Paiva, V. (2012b). A dimensão psicossocial do cuidado. In V. Paiva, G. Calazans, \& A. Segurado (Orgs.), Coletânea: Vulnerabilidade e Direitos Humanos. Prevenção e promoção da saúde: Vol. 2. Entre indivíduos e comunidades (2. ed., pp. 41-72). Curitiba, PR: Juruá.

Paiva, V., Ayres, J. R., \& Franca, I., Jr. (2004, January). Expanding the flexibility of normative patterns in youth sexuality and prevention programs. Sexuality Research \& Social Policy, 1(1).

Parker, R., \& de Camargo, K., Jr. (2000). Pobreza e HIV/AIDS: Aspectos antropológicos e sociológicos. Cadernos de Saúde Pública, 16(1), 89-102.

Pinheiro, R., \& Lopes, T. C. (Orgs.). (2010). Ética, técnica e formação: As razões do cuidado como direito à saúde. Rio de Janeiro, RJ: Centro de Estudos e Pesquisa em Saúde Coletiva.

Pinheiro, R., \& Mattos, R. A. (2006). Cuidado: As fronteiras da integralidade. Rio de Janeiro, RJ: Centro de Estudos e Pesquisa em Saúde Coletiva.

Pupo, L. R. (2012). As dimensões teórica, técnica e ético-operacional: Multidimensionalidade da ação preventiva e de cuidado à saúde. In V. Paiva, G. Calazans, \& A. Segurado (Orgs.), Coletânea: Vulnerabilidade e Direitos Humanos. Prevenção e promoção da saúde: Vol. 2. Entre indivíduos e comunidades (2. ed., pp. 41-72). Curitiba, PR: Juruá.

Rother, E. T. (2007). Revisão sistemática X revisão narrativa. Acta Paulista de Enfermagem, 20(2), 5-6. Recuperado en 26 de setembro, 2013, de http://www.scielo.br/ scielo.php?script $=$ sci_arttext\&pid $=\mathrm{S} 0103$ $-21002007000200001 \& \operatorname{lng}=$ en $\& \operatorname{tlng}=$ pt. $10.1590 / \mathrm{S} 0103-21002007000200001$

Santos, B. S. (2003). Reconhecer para libertar: Os caminhos do cosmopolitismo multi-cultural. Rio de Janeiro, RJ: Civilização Brasileira.

Santos, F. M., \& Jacó-Vilela, A. M. (2009, maio/ ago.). O psicólogo no hospital geral: Estilos e coletivos de pensamento. Paidéia (Ribeirão Preto), 19(43), 189-197.

Scott, J. W. (1995, jul./dez.). Gênero: Uma categoria útil de análise histórica. Educação \& Realidade, 20(2), 71-99. (Revisão de T. T. da Silva a partir de Gender and the Politics of History, de J. W. Scott, 1988, pp. 28-50, New York: Columbia University Press) 
Seffner, F., Paiva, V., \& Pupo, L. (2012). Educação em saúde e emancipação: Explorando possibilidades da prevenção no quadro dos direitos humanos In V. Paiva, L. Rivero Pupo, \& F. Seffner (Orgs.), Coletânea: Vulnerabilidade e Direitos Humanos - Prevenção e promoção da saúde: Vol. 3. Pluralidade de vozes e inovação de práticas (pp. 253-268.). Curitiba, PR: Juruá.

Silva, C. G., Santos, A. O., Licciardi, D. C., \& Paiva, V. (2008). Religiosidade, juventude e sexualidade: Entre a autonomia e a rigidez. Psicologia em Estudo, 13(4).
Simon, W., \& Gagnon, J. H. (1969). On psychosocial development. In D. A. Goslin (Ed.), Handbook of Socialization Theory and Research (pp. 733752). Chicago, IL: Rand McNally.

Spink, M. J. (2013). Psicologia Social e Saúde. Práticas, saberes e sentidos. Petrópolis, RJ: Vozes

Tunala, G. L. (2012). Modelos teóricos como subsídios da prática da promoção da saúde em DST/ Aids no quadro da vulnerabilidade e dos direitos humanos. In V. Paiva, G. Calazans, \& A. Segurado (Orgs.), Coletânea: Vulnerabilidade e Direitos Humanos. Prevenção e promoção da saúde: Vol. 2. Entre indivíduos e comunidades (2. ed.). Curitiba, PR: Juruá. 